\title{
Ratchet device with broken friction symmetry
}

Norden, Bengt; Zolotaryuk, Yaroslav; Christiansen, Peter Leth; Zolotaryuk, Alexander

\section{Published in:}

Applied Physics Letters

Link to article, DOI:

$10.1063 / 1.1468900$

Publication date:

2002

Document Version

Publisher's PDF, also known as Version of record

Link back to DTU Orbit

Citation (APA):

Norden, B., Zolotaryuk, Y., Christiansen, P. L., \& Zolotaryuk, A. (2002). Ratchet device with broken friction symmetry. Applied Physics Letters, 80(14), 2601-2603. https://doi.org/10.1063/1.1468900

\section{General rights}

Copyright and moral rights for the publications made accessible in the public portal are retained by the authors and/or other copyright owners and it is a condition of accessing publications that users recognise and abide by the legal requirements associated with these rights.

- Users may download and print one copy of any publication from the public portal for the purpose of private study or research.

- You may not further distribute the material or use it for any profit-making activity or commercial gain

- You may freely distribute the URL identifying the publication in the public portal

If you believe that this document breaches copyright please contact us providing details, and we will remove access to the work immediately and investigate your claim 


\title{
Ratchet device with broken friction symmetry
}

\author{
Bengt Nordén \\ Department of Physical Chemistry, Chalmers University of Technology, S-412 96 Gothenburg, Sweden
}

Yaroslav Zolotaryuk, ${ }^{\text {a) }}$ Peter L. Christiansen, and Alexander V. Zolotaryuk

Section of Mathematical Physics, IMM, Technical University of Denmark, DK-2800 Lyngby, Denmark

(Received 26 July 2001; accepted for publication 12 February 2002)

\begin{abstract}
An experimental setup (gadget) has been made for demonstration of a ratchet mechanism induced by broken symmetry of a dependence of dry friction on external forcing. This gadget converts longitudinal oscillating or fluctuating motion into a unidirectional rotation, the direction of which is in accordance with given theoretical arguments. Despite the setup being three dimensional, the ratchet rotary motion is proved to be described by one simple dynamic equation. This kind of motion is a result of the interplay of friction and inertia. () 2002 American Institute of Physics.

[DOI: $10.1063 / 1.1468900]$
\end{abstract}

As described originally by Smoluchowski ${ }^{1}$ and noted by Feynman, ${ }^{2}$ a Brownian particle can undergo net transport on a potential energy surface, even if it is externally driven by oscillating or fluctuating forces of zero average. ${ }^{3}$ These socalled Brownian ratchets have attracted considerable attention because of their description of molecular motors ${ }^{4}$ and other technological devices. ${ }^{5-9}$ Among these, the ratchet model that contains finite inertia, ${ }^{10}$ represents a considerable technical challenge because under certain conditions it admits a chaotic behavior, which has to be suppresed in technological applications.

The fundamental condition for the rectified transport to occur is that certain symmetries, associated with spatial or time reflection, are broken. ${ }^{11}$ On the other hand, a similar symmetry breaking can also be achieved parametrically, when at least one of the system parameters depends asymmetrically on the velocity or external forcing. More precisely, let us consider the dynamic equation for a particle moving in a periodic potential $U(x)$, with $x=x(t)$ being a coordinate of the particle, generalized to include some additional dynamic properties such as nonlinear friction, dependence on external forcing, etc.,

$$
\ddot{x}+U^{\prime}(x)+\gamma(f) \sigma(\dot{x}) / \dot{x}=f .
$$

Here the friction is supposed to depend on an external force $f(t)$ with zero average and the function $\sigma=\sigma(\dot{x})$ describes the type of friction. Following the symmetry arguments, ${ }^{11}$ one can classify the following three particular types of Eq. (1), each of which admits ratchet dynamics.

(i) The friction is a symmetric function with respect to an external force $[\gamma(-f)=\gamma(f)]$ and its dependence on the velocity is linear $\left(\sigma=\dot{x}^{2}\right)$. The rectification in this case occurs either due to broken symmetries in space $[U(-x)$ $\neq U(x)]$ or in time (e.g., harmonic mixing), ${ }^{3,11}$ except for supersymmetric ratchets. ${ }^{12}$

(ii) The periodic potential is absent $[U(x) \equiv 0]$ and $\gamma(-f)=\gamma(f)$. The rectification occurs in the case when both nonlinearity of $\sigma$ (e.g., if $\sigma=\dot{x}^{2}+\dot{x}^{4}$ ) and broken time symmetry (e.g., due to harmonic mixing $)^{5}$ are present.

${ }^{a)}$ Electronic mail: yz@imm.dtu.dk (iii) The periodic potential is absent $[U(x) \equiv 0]$, the function $\sigma(\dot{x})$ does not necessarily result in a nonlinear friction term in the equation of motion (1), and the time symmetry is not broken. The rectification can occur due to broken symmetry of the force [if $\gamma(-f) \neq \gamma(f)]$ or velocity [if $\sigma(-\dot{x}) \neq \sigma(\dot{x})]$ dependent friction.

In this letter, we focus on the last possibility, when $\sigma(\dot{x})=|\dot{x}|$ (dry friction). In this case, if the system is sufficiently sophisticated, a ratchet motion can occur through a broken symmetry of the dependence of friction on an external driving force. To support the idea of the ratchet as a result of broken friction symmetry, we have constructed an experimental device (see its photograph and schematic sketch in Figs. 1 and 2, respectively) - a mechanical diode that changes the friction coefficient, while an external force is applied to the system, resulting in a biased rotational motion.

As depicted in Figs. 1 and 2, the setup consists of two massive plates (weights) $A$ and $B$ with masses $M_{A}$ and $M_{B}$, which are connected by two lateral springs 1 and 2, so that their geometric arrangement mimics a right-handed helical

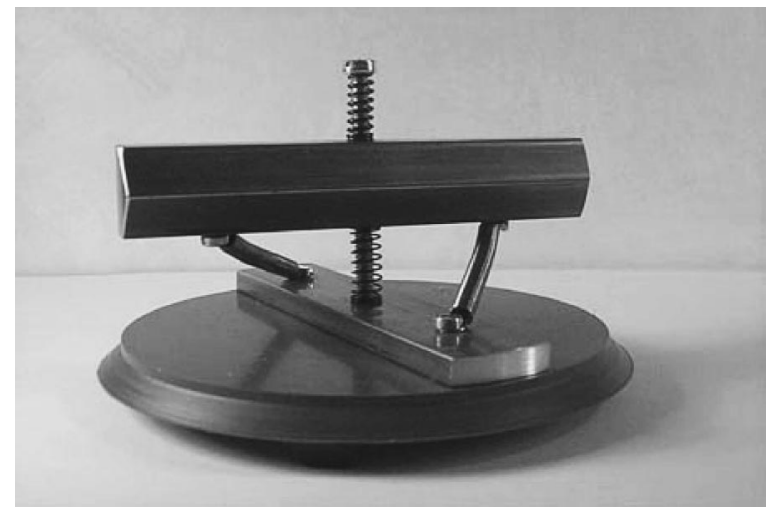

FIG. 1. The experimental setup that consists of two (upper and lower) helically rotating coupled plates. The supporting bottom plate creates friction for the lower plate. A driving oscillating or fluctuating force is applied to the upper plate, e.g., by hitting it slightly from various directions. Colored noise was created by the sound from a fog horn, acting on a horizontal membrane glued on top of the upper plate. In all cases of forcing vertically (from above or below), the helical system was observed to rotate only clockwise when viewed from above. 


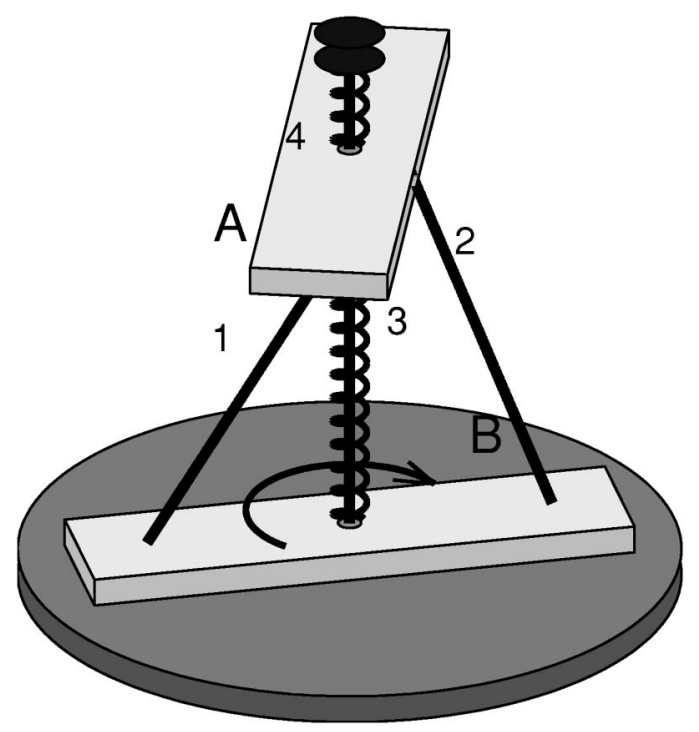

FIG. 2. Schematic sketch of the setup: the two lateral springs (acting as elastic rods) 1 and 2 are shown by thick solid lines.

structure. In experiments with this helicity, the device shows unambiguously the rotary ratchet motion directed clockwise, when viewed from above (shown with the arrow in Fig. 2), whereas the left-handed helicity implies the counterclockwise rotation. In other words, the change of direction of springs 1 and 2 does not affect the problem of rectification, changing only the direction of rectified motion. Next, it is important that springs 1 and 2 are very soft to bend, but hard to compress or stress (being in fact elastic rods). Weight $A$ is resting on weight $B$ with a lower vertical spring (3) in between, while an upper vertical spring (4), for which the upper end is fixed, controls the pressure of plate $B$ on a supporting plate. Therefore, the vertical distance between plates $A$ and $B$ is fixed only by vertical springs 3 and 4 , and the lateral springs do not participate in the force balance. The ends of the lateral springs are attached to the plates rigidly, whereas the vertical springs are allowed to slide freely on the surfaces of the plates when they rotate.

In equilibrium, both springs 3 and 4 are little pressed, and this construction allows us to exclude effectively the role of gravity in experiments. Indeed, when the gadget is oriented, e.g., horizontally, plate $A$ will be found in another (shifted) equilibrium position, but still being pressed to the supporting plate, resulting in a nonzero friction. Therefore, gravity is not essential for the existence of a ratchet motion and both weights $A$ and $B$ can be considered as masses. Instead, the inertia of plate $A$ is crucial for the sliding rotation of plate $B$ on the supporting plate. In a rotation around the vertical axis, the friction of weight $B$ depends on the current vertical position of weight $A$; that friction is larger as the weight (force) on spring 3 is larger, so that when an external oscillating or fluctuating force is applied on weight $A$, the friction in a rotation of weight $B$ sliding on the supporting plate depends strictly on the force applied to weight $A$. The pair of lateral springs (elastic rods) converts the oscillating or fluctuating normal force applied on weight $A$ into a force changing the angle between the rotating plates. Because the friction coupling between the $A$ rotation and the $B$ rotation is larger when spring 3 is contracted than when it is Downloaded 05 Mar 2010 to 192.38.67.112. Redistribution subject extended, we get a rectification as the lateral springs transfer a normal motion into a rotation of plate $B$.

Let $R_{0}$ be the radius of the helical backbone of the setup. The equilibrium state of the system is given by the dimensionless (measured in units of $R_{0}$ ) vertical distance $h$ and the mismatch angle $\phi<\pi / 2$. Under different motions of plates $A$ and $B$, the length of springs 1 and 2 , given by $l$ $=\sqrt{h^{2}+2(1-\cos \phi)}$, practically does not change and therefore they create a constraint in the system. The supporting plate in the setup (see Figs. 1 and 2) generates a sliding friction for a rotary motion of plate $B$. This friction depends on the normal response force $N_{B}$ directed upwards and created by the supporting plate. In its turn, this response depends on how spring 3 is pressed (or stretched), and this dependence is governed by an external normal force $F_{n}(t)$, acting from plate $A$ through spring 3 as well as through the lateral springs, acting as a constraint. Any tangential forcing that exceeds the friction of rest, results in a rotation of plate $B$.

One of the equations of motion can be derived for the angular variables $\theta_{A}(t)$ and $\theta_{B}(t)$, instantaneous deviations of plates $A$ and $B$ from their equilibria. We denote the moments of inertia by $a M_{A} R_{0}^{2}$ and $b M_{B} R_{0}^{2}$, with $a$ and $b$ being (dimensionless) geometric form factors for plates $A$ and $B$, respectively. Since the lateral springs are soft to bend, the interaction of $A$ and $B$ through these springs in the vertical direction can be ignored. This technical point essentially simplifies the full system of dynamic equations, which in general take a very complicated form. Therefore, in this setup, one can account for only a tangential response force $T_{t}$ that appears due to the constraint created by the lateral springs. In general, except for the force $T_{t}$, an external tangential force $F_{t}$ may be applied, so that the tangential equation of motion for plate $A$ is $a M_{A} R_{0} \ddot{\theta}_{A}=T_{t}+F_{t}$. The second equation that governs the friction dynamics of plate $B$ can be written in the form $b M_{B} R_{0} \ddot{\theta}_{B}+G\left(\dot{\theta}_{B}, N_{B}\right) /\left(R_{0} \dot{\theta}_{B}\right)=-T_{t}$, where the dissipation function $G$ for the lower plate depends on the angular velocity $\dot{\theta}_{B}$ and the response force $N_{B}$, acting from the side of the supporting plate. Next, the response force $T_{t}$ can be excluded from these two equations of motion and, as a result, we obtain one tangential equation for the angle variables $\theta_{A}$ and $\theta_{B}$ :

$$
a M_{A} \ddot{\theta}_{A}+b M_{B} \ddot{\theta}_{B}+G\left(\dot{\theta}_{B}, N_{B}\right) /\left(R_{0}^{2} \dot{\theta}_{B}\right)=F_{t} / R_{0} .
$$

The equation of motion that describes the vertical dynamics of plate $A$ driven by the normal force $F_{n}(t)$ reads

$$
M_{A} \ddot{z}+\eta_{A} M_{A} \dot{z}+K V^{\prime}(z)=F_{n} / R_{0},
$$

where $z$ is the displacement of plate $A$ from its vertical equilibrium position given by springs 3 and 4 (and weight $A$ if the gadget is oriented vertically). The function $V(z)$, with a minimum at $z=0$, describes the strain energy of the vertical springs. The string parameters $\eta_{A}$ and $K$ stand for the friction and stiffness, respectively.

Finally, the last equation of motion results from the constraint imposed on plates $A$ and $B$ by the stiff lateral springs (acting in fact as rods), leading to a geometric relation between the variables $\theta_{A}, \theta_{B}$, and $z$. Since the length of the lateral springs in our setup is practically unchanged (because they are too hard to compress or stress), one finds in the AIP license or copyright; see http://apl.aip.org/apl/copyright.jsp 


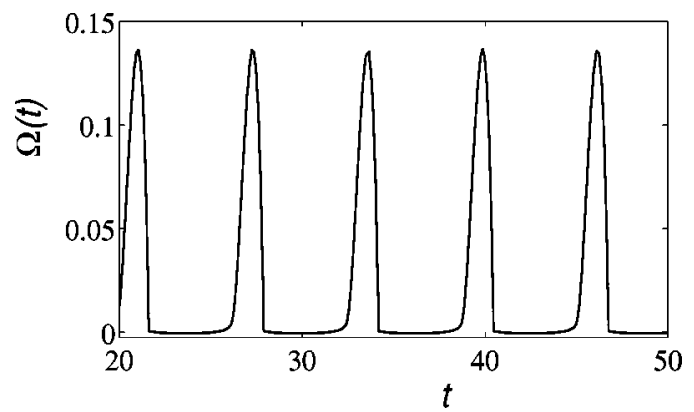

FIG. 3. Rotational velocity $\Omega(t)$ in the case of the rational friction dependence $\gamma(f)=\gamma_{0}\left(z_{1}-f\right) /\left(f-z_{2}\right)$ and the driving force $f(t)=f_{0} \sin (\omega t)$, computed numerically for $\gamma_{0}=1, z_{1}=-z_{2}=1, f_{0}=1 / 2$, and $\omega=1$.

linear approximation the relation $z=h^{-1}\left(\theta_{A}-\theta_{B}\right) \sin \phi$. Using this equation, the variable $\theta_{A}$ can be eliminated in Eq. (2), leading to the equation

$$
\Omega+G\left(\Omega, N_{B}\right) /\left(M R_{0}^{2} \Omega\right)=-\mu \ddot{z}
$$

with respect to the angular velocity $\Omega \equiv \dot{\theta}_{B}$ of plate $B$. Here $M=a M_{A}+b M_{B}$ and $\mu=a h M_{A} /(M \sin \phi)$ are system parameters. As follows from Eq. (2), the tangential force $F_{t}$ does not affect the rectification of rotary motion and therefore it is omitted in Eq. (4). Without loss of generality, one can write $G\left(\Omega, N_{B}\right)=M_{B} R_{0}^{2} \Gamma\left(N_{B}\right) \sigma(\Omega)$, where $\sigma(-\Omega)$ $=\sigma(\Omega)$ and the friction coefficient $\Gamma$ obviously increases with $N_{B}$. In our gadget, we deal with dry friction and therefore one can put $\sigma(\Omega)=|\Omega|$, neglecting more sophisticated dependences, which are still subject to controversy. ${ }^{13,14}$

As mentioned earlier, the response $N_{B}$ depends on how spring 3 is stretched or compressed, i.e., on the displacement $z$ of plate $A$ from its equilibrium. More precisely, the dependence $N_{B}=N_{B}(z)$ is given by the equation $N_{B}$ $=-K R_{0} V^{\prime}\left(z-z_{1}\right)$ if $z \leqslant z_{1}$ and $N_{B}=0$ if $z>z_{1}$, where $z_{1}$ $>0$ is the distance, at which spring 3 becomes completely unpressed. Therefore, the function $\bar{\Gamma}(z) \equiv \Gamma\left[N_{B}(z)\right]$ must decrease with $z$ and become zero for all $z>z_{1}$. On the other hand, when plate $B$ is strongly pressed by spring 3 to the supporting plate, the rotation is entirely supressed. This occurs for $z<z_{2}$, where $z_{2}$ is a critical value (friction at rest), below which the friction becomes infinite. The function $\bar{\Gamma}(z)=\bar{\Gamma}_{0} \exp \left[\alpha /\left(z-z_{1}\right)\right]\left(z-z_{2}\right)^{-1}, \alpha>0, \bar{\Gamma}_{0}>0$, defined on the interval $z_{2}<z<z_{1}$, is mostly appropriate to describe the friction dependence on the displacement $z$ because it smoothly approaches zero as $z \rightarrow z_{1}$, being infinitely differentiable at this point. Obviously, this is not a unique choice; in general, any monotonically decreasing function, like an exponential behavior ${ }^{14}$ or the rational function $\bar{\Gamma}(z)=\bar{\Gamma}_{0}\left(z_{1}\right.$ $-z) /\left(z-z_{2}\right)$, can be chosen for a qualitative analysis of the friction dynamics.

In the particular case of a sinusoidal force $F_{n}(t)$ with a frequency $\omega$, the steady-state solution (trajectory or attractor) of Eq. (3) is also a sinusoidal function with the amplitude $z_{0}$ being proportional to the force amplitude $F_{0} \cdot{ }^{15}$ Next, we denote the right-hand side of Eq. (4) by $f(t)$ $\equiv \mu z_{0} \omega^{2} \sin (\omega t)$, acting as an external force on plate $B$. Then Eq. (4) can be rewritten in the form

$$
\dot{\Omega}+\operatorname{sgn}(\Omega) \gamma(f)=f,
$$

with a dimensionless friction coefficient $\gamma$ depending on the force $f(t)$.

As expected intuitively, for any nonincreasing, but necessarily decreasing at least in some neighborhood of the point $f=0$ in the domain of the function $\gamma(f)$, the global (average) velocity $\langle\Omega(t)\rangle$ can be proved rigorously to be positive and this means that plate $B$ rotates clockwise as shown in Fig. 2. In the particular case of the rational function, the steady-state solution to Eq. (5) was found numerically. As shown in Fig. 3, after starting numerical simulations, the solution $\Omega(t)$ approaches the steady state very fast. This figure clearly demonstrates that plate $B$ practically never steps backwards and this behavior is indeed observed visually in experiments with our gadget.

In conclusion, we have discussed the ratchet mechanism, which can occur due to broken friction symmetry in the equation of motion (1) or (4) and seems to be important for applications. In particular, combination of self-propulsion theories ${ }^{16}$ with the asymmetric dependence of the friction on (internal or external) forcing or velocity can gain an insight into physics of microbiological motility. ${ }^{17}$

The authors would like to acknowledge partial support from the European Union under the INTAS Grant No. 970368 and the RTN Project No. LOCNET HPRN-CT-199900163. The authors thank A. C. Scott, A. K. Vidybida, and V. N. Ermakov for stimulating and helpful discussions.

\footnotetext{
${ }^{1}$ M. von Smoluchowski, Phys. Z. 13, 1069 (1912).

${ }^{2}$ R. P. Feynman, R. B. Leighton, and M. Sands, The Feynman Lectures on Physics (Addison-Wesley, Reading, MA, 1966), Vol. 1, Chap. 46.

${ }^{3}$ For the latest review on this problem see the preprint by P. Reimann, cond-mat/0010237.

${ }^{4}$ R. D. Astumian, Science 276, 917 (1997); F. Jülicher, A. Ajdari, and J. Prost, Rev. Mod. Phys. 69, 1269 (1997).

${ }^{5}$ A. K. Vidybida and A. A. Serikov, Phys. Lett. A 108, 170 (1985).

${ }^{6}$ J. Rousselet, L. Salome, A. Ajdari, and J. Prost, Nature (London) 370, 446 (1994).

${ }^{7}$ I. Zapata, R. Bartussek, F. Sols, and P. Hänggi, Phys. Rev. Lett. 77, 2292 (1996).

${ }^{8}$ F. Marchesoni, Phys. Lett. A 237, 126 (1998).

${ }^{9}$ M. Borromeo and F. Marchesoni, Phys. Lett. A 249, 199 (1998); Appl. Phys. Lett. 75, 1024 (1999).

${ }^{10}$ P. Jung, J. G. Kissner, and P. Hänggi, Phys. Rev. Lett. 76, 3436 (1996).

${ }^{11}$ S. Flach, O. Yevtushenko, and Y. Zolotaryuk, Phys. Rev. Lett. 84, 2358 (2000).

${ }^{12}$ P. Reimann, Phys. Rev. Lett. 86, 4992 (2001).

${ }^{13}$ T. Baumberger, F. Heslot, and B. Perrin, Nature (London) 367, 544 (1994)

${ }^{14}$ V. Zaloj, M. Urbakh, and J. Klafter, Phys. Rev. Lett. 82, 4823 (1999).

${ }^{15}$ See, e.g., L. D. Landau and E. M. Lifshitz, Mechanics (Pergamon, Oxford, 1960).

${ }^{16}$ K. M. Ehlers, A. D. T. Samuel, H. C. Berg, and R. Montgomery, Proc. Natl. Acad. Sci. U.S.A. 93, 8340 (1996).

${ }^{17}$ J. B. Waterbury, J. M. Willey, D. G. Franks, F. W. Valois, and S. W.
} Watson, Science 230, 74 (1985). 